\title{
Dwarf Nova Oscillations and Quasi-Periodic Oscillations: Extension of the Two-QPO Diagram of X-Ray Binaries, and a new kind of DNO
}

\author{
Brian Warner and Patrick A. Woudt \\ Department of Astronomy, University of Cape Town, Rondebosch 7700, \\ South Africa
}

\begin{abstract}
Seventeen examples are given of Cataclysmic Variable (CV) stars possessing both Dwarf Nova Oscillations (DNOs) and Quasi-Periodic Oscillations (QPOs). These form an extension of the X-Ray Two-QPO correlation to frequencies three orders of magnitude lower. We draw attention to the existence of a second type of DNO in CVs, which is probably caused by magnetically channelled accretion onto the white dwarf.
\end{abstract}

\section{Introduction}

We reported at the August 2001 Göttingen meeting on CVs that VW Hyi in outburst has Dwarf Nova Oscillations (DNOs) and Quasi-Periodic Oscillations (QPOs) that keep an approximately constant period ratio of $\sim 16$ as they vary in period by a factor of two during the late stages of outburst (Warner \& Woudt 2002a; see also Woudt \& Warner 2002). Interpreting the DNOs and QPOs in VW Hyi as analogues of the high and low frequency QPOs observed in X-Ray binaries, we showed that VW Hyi lies on an extension to low frequencies of the two-QPO correlation in X-Ray binaries found by Psaltis, Belloni \& van der Klis (1999). An important addition to this correlation was made by Mauche (2002), who found that the X-Ray DNOs and QPOs in SS Cyg also fall on this relationship, which helps to close the gap between the CVs and the X-Ray binaries.

One aspect of QPOs in CVs is that double DNOs are sometimes observed, and their frequency difference is equal to the QPO frequency (Woudt \& Warner 2002 ) - which we interpret as the DNO rotating beam reprocessed off a progradely travelling wave in the inner disc (Warner \& Woudt 2002b). Therefore, even if a QPO is not directly observed in a light curve, a proxy for it can be found when double DNOs are observed. 


\section{The Two-QPO Diagram for CVs and X-Ray Binaries}

Armed with this knowledge we have searched the literature for examples of the simultaneous appearance of DNOs and QPOs, or their proxies. Table 1 lists the results of the search.

Table 1. DNOs and QPOs in CVs (periods in seconds).

\begin{tabular}{|c|c|c|c|}
\hline Star & DNOs & QPOs & References \\
\hline V3885 Sgr & $29.08 \quad 30.1$ & [819] & Hesser, Lasker \& Osmer 1974 \\
\hline WZ Sge & 27.8728 .95 & 742 & Warner \& Woudt 2002b \\
\hline SS Cyg & 7.7 & 83 & Mauche 2002 \\
\hline V436 Cen & 19.6 & 475 & Warner \& Woudt 2002b \\
\hline & 19.4520 .29 & [470] & Warner \& Woudt (unpublished) \\
\hline V2051 Oph & 28.0629 .77 & {$[489]$} & Steeghs et al. 2001 \\
\hline UX UMa & 29 & 650 & Nather \& Robinson 1974 \\
\hline IX Vel & $\sim 28$ & $\sim 500$ & $\begin{array}{l}\text { Warner et al. } 1985 \\
\text { Williams \& Hiltner } 1984\end{array}$ \\
\hline TY PsA & 27 & 245 & Warner et al. 1989 \\
\hline GK Per & 351 & $\sim 5000$ & Morales-Rueda et al. 1996,1999 \\
\hline RX And & 35.7 & $\sim 1200$ & Szkody 1976 \\
\hline SW UMa & 22.3 & $\sim 300$ & Robinson et al. 1987 \\
\hline RU Peg & $2.94 ?$ & 50 & Patterson et al. 1977 \\
\hline
\end{tabular}

Notes: values in square brackets are deduced from double DNOs. In RU Peg the observed DNO period is $11.1 \mathrm{~s}$, which is probably a beat with the integration duration.

In addition, we have four CVs in which we have recently observed DNOs and QPOs; these are listed in Table 2.

Table 2. Newly observed DNOs and QPOs (periods in seconds).

\begin{tabular}{|c|c|c|c|c|}
\hline Star & Type & & DNOs & QPOs \\
\hline WX Hyi & Dwarf Nova & & 12.1 & 190 \\
\hline VZ Pyx & Dwarf Nova & & 23.86 & 390.5 \\
\hline EC 2117-54 & Nova-like & 22.11 & 23.28 & 470 \\
\hline HX Peg & Dwarf Nova & & 112.28 & 1800 \\
\hline
\end{tabular}

The addition of these data, obtained from observing the literature and observing the sky, to the Two-QPO diagram produces the correlation shown in Figure 1. It can be seen that the CV DNO and QPO observations lie on an 


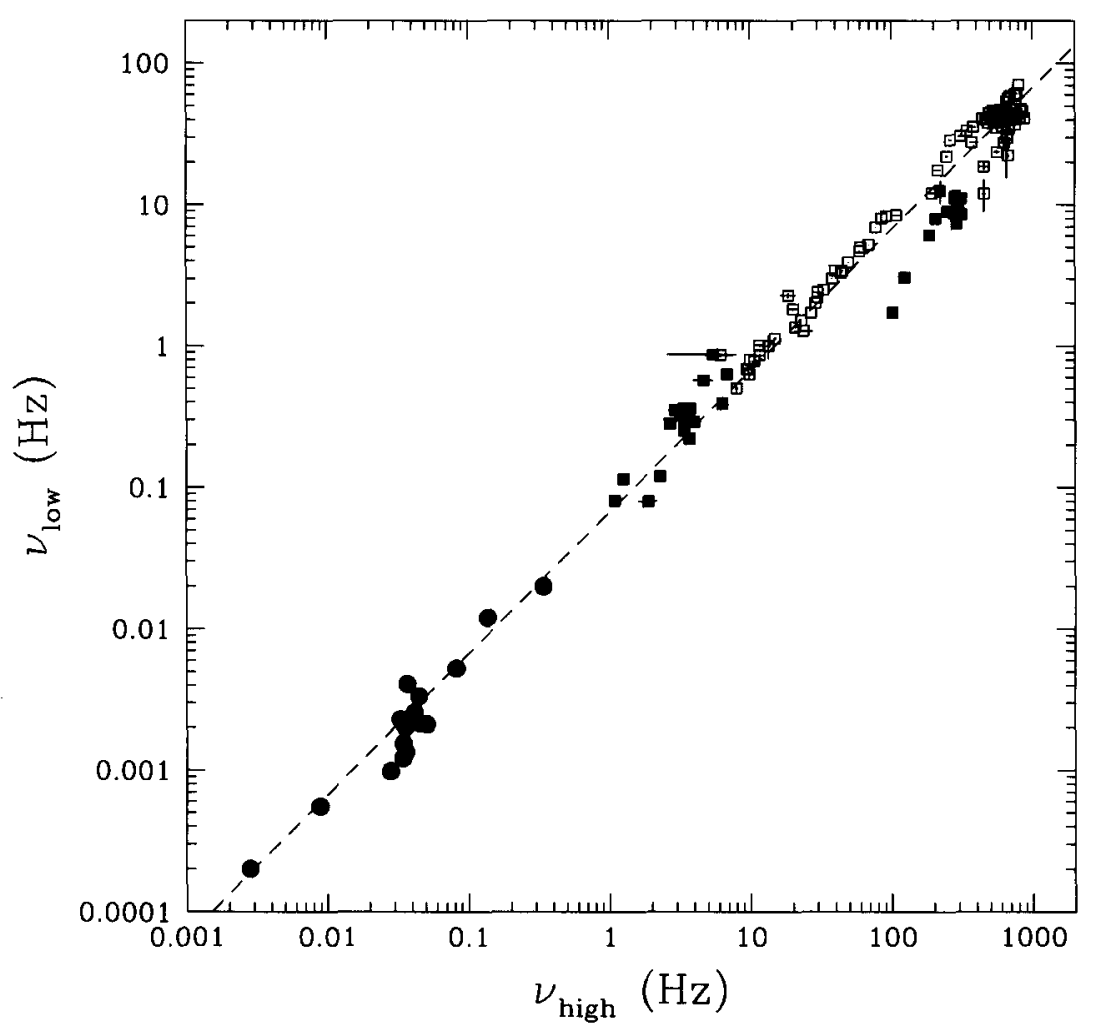

Figure 1. The Two-QPO diagram for X-Ray Binaries (filled squares: black hole binaries; open squares: neutron star binaries) and $17 \mathrm{CVs}$ (filled circles). The X-Ray binary data are from Belloni, Psaltis \& van der Klis (2002) and were kindly provided by T. Belloni. The dashed line marks $\mathrm{P}_{Q P O} / \mathrm{P}_{D N O}=15$.

extension of the X-Ray Binary relationship to frequencies nearly three orders of magnitude lower. The essence of both the CV and the X-Ray correlations is that $\mathrm{P}_{Q P O} \sim 15 \mathrm{P}_{D N O}$.

Adopting a magnetically controlled accretion model for DNOs (Paczynski 1978; Warner 1995; Warner \& Woudt 2002b), the DNO period is clearly related to the Keplerian period at the magnetically truncated inner edge of the disc. A number of recent papers modelling accretion into magnetospheres find quasi-periodic accretion or oscillations (e.g. Uzdensky 2002; Titarchuk \& Wood 2002); a quantitative model by Goodson, Bohm \& Winglee (1999) deduces $\mathrm{P}_{Q P O} / \mathrm{P}_{D N O} \sim 100 / 2 \pi \sim 16$. 


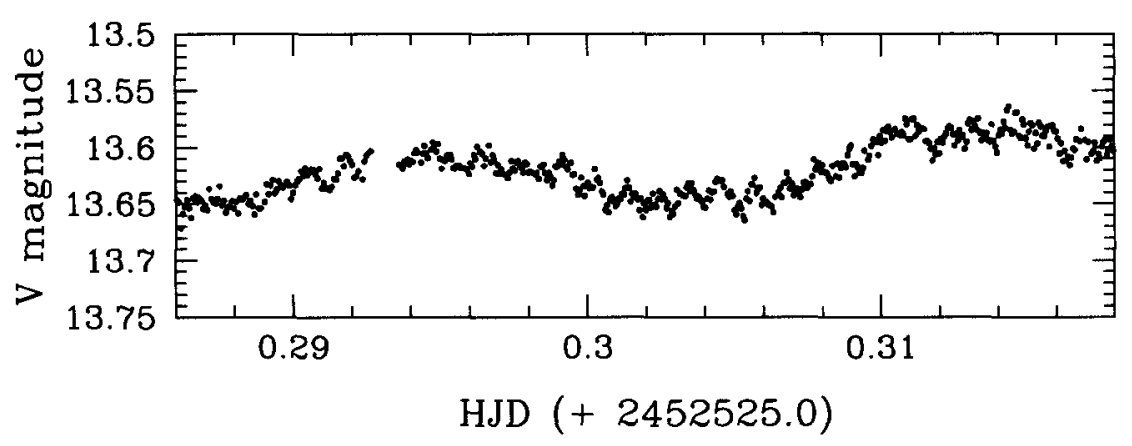

Figure 2. Longer period DNOs observed in EC 2117-54

\section{A New kind of DNO}

During the literature search for DNOs and QPOs we noticed that there are occasionally additional DNOs listed that do not fall into the above category - indeed, several CVs show two sets of DNOs simultaneously. Furthermore, the second kind of DNOs behave very differently from the 'classical' DNOs, in that their periods are relatively independent of outburst state, and they can appear during quiescence of dwarf novae (which, apart from the apparently more strongly magnetic systems WZ Sge and GK Per, normal DNOs do not).

The new type of DNOs, which we will refer temporarily as 'longer period DNOs' - lpDNOs, are demonstrated in the following stars:

VW Hyi: In a superoutburst of VW Hyi observed in 1975 Haefner, Schoembs \& Vogt (1977, 1979, see also Schoembs 1977) found an 80 s DNO which persisted for nine nights and changed only slightly and erratically in period although the star decreased in brightness from mag 10.6 to 13.7. We have also seen occasional DNOs near $88 \mathrm{~s}$ in VW Hyi. The normal DNO periods in VW Hyi range from $14 \mathrm{~s}$ at maximum of outburst to $40 \mathrm{~s}$ near return to quiescence (Woudt \& Warner 2002).

EC 2117-54: This newly discovered nova-like CV, from the EdinburghCape Survey, possesses normal (including double) DNOs near $23 \mathrm{~s}$ and QPOs near $450 \mathrm{~s}$, but in addition frequently shows lpDNOs near $94 \mathrm{~s}$ (see Figure 2). Thus EC 2117-54 has a suite of oscillations that closely resembles that of VW Hyi in outburst.

SS Cyg: Robinson \& Nather (1979) and Patterson (1981) observed what they described as QPOs in the range $32-36 \mathrm{~s}$ in two different outbursts of SS Cyg, at the same time $\sim 10$ s normal DNOs were present.

HT Cas and AH Her: Patterson (1981) observed $~ 100 \mathrm{~s}$ QPOs in HT Cas and $\mathrm{AH}$ Her in outburst, where the normal DNOs are $\sim 20 \mathrm{~s}$ and $\sim 25 \mathrm{~s}$ respectively. The HT Cas oscillations were also often seen during quiescence.

OY Car: We have observed strong $\sim 50$ s oscillations during one observational run on OY Car at quiescence. This star has normal DNOs of $18-28 \mathrm{~s}$ during outbursts. 
For a possible interpretation of the lpDNOs we turn to measurements of $v \sin i$ of the white dwarf primaries. In VW Hyi Sion et al. $(1996,2001)$ have found projected rotational velocities in the range $300-500 \mathrm{~km} \mathrm{~s}^{-1}$. For $i=$ $65^{\circ}$ and a primary mass of $0.6 \mathrm{M}_{\odot}$ this gives a rotation period of $120-165 \mathrm{~s}$. The observed lpDNO may therefore be associated with the bulk rotation of the primary (as opposed to the rotation of the equatorial belt in the case of the normal DNOs - Warner \& Woudt 2002a,b), with two-pole magnetic accretion. A similar result is obtained for SS Cyg, with $v \sin i=300 \mathrm{~km} \mathrm{~s}^{-1}$ (Sion 1999), $i=50^{\circ}$ and $\mathrm{M}=1.2 \mathrm{M}_{\odot}$, which give a primary rotation period $\sim 63 \mathrm{~s}$.

We suggest, therefore, that the lpDNOs result from accretion controlled by the magnetic field of the primary itself, whereas the normal DNOs come from accretion onto the equatorial belt where a stronger field is generated by differential rotation.

Acknowledgments. BW is supported by funds from the University of Cape Town; PAW is supported by strategic funds made available to BW by the University of Cape Town and by the National Research Foundation. We thank T. Belloni for kindly providing us with the X-Ray Binary data shown in Figure 1.

\section{References}

Belloni, T., Psaltis, D., \& van der Klis, M. 2002, ApJ, 572, 392

Goodson, A.P., Bohm, K.-H., \& Winglee, R.M. 1999, ApJ, 524, 142

Haefner, R., Schoembs, R., \& Vogt, N. 1977, A\&A, 61, 37L

Haefner, R., Schoembs, R., \& Vogt, N. 1979, A\&A, 77, 7

Hesser, J.E., Lasker, B.M., \& Osmer, P.S. 1974, ApJ, 189, 315

Mauche, C. 2002, ApJ, 580, 423

Morales-Rueda, L., Still, M.D., \& Roche, P. 1996, MNRAS, 283, L58

Morales-Rueda, L., Still, M.D., \& Roche, P. 1999, MNRAS, 306, 753

Nather, R.E., \& Robinson, E.L. 1974, ApJ, 190, 637

Paczynski, B. 1978, in "Nonstationary Evolution of Close Binaries", ed. A. Zytkow (Polish Sci. Publ.: Warsaw), 89

Patterson, J, 1981, ApJS, 45, 517

Patterson, J., Robinson, E.L., \& Nather, R.E. 1977, ApJ, 214, 144

Psaltis, D., Belloni, T., \& van der Klis, M. 1999, ApJ, 520, 262

Robinson, E.L., \& Nather, R.E. 1979, ApJS, 39, 461

Robinson, E.L., Shafter, A.W., Hill. J.A., \& Wood, M.A. 1987, ApJ, 313, 772

Schoembs, R. 1977, IAU Coll. No. 42, p. 218

Sion, E.M. 1999, PASP, 111, 532

Sion, E.M., Cheng, F.-H., Huang, M., Hubeny, I., \& Szkody, P. 1996, ApJ, 471, L41

Sion, E.M., Cheng, F.-H., Szkody, P. (+7 co-authors) 2001, MNRAS, 323, 484

Steeghs, D., O'Brien, K., Horne, K., Gomer, R., \& Oke, J.B. 2001, MNRAS, 323,484 
Szkody, P. 1976, ApJ, 207, 190

Titarchuk, L., \& Wood, K. 2002, ApJ, 577, L23

Uzdensky, D.A. 2002, ApJ, 572, 432

Warner, B. 1995, ASP Conf. Ser. 85, 343

Warner, B., \& Woudt, P.A. 2002a, ASP Conf. Ser. 261, 406

Warner, B., \& Woudt, P.A. 2002b, MNRAS, 335, 84

Warner, B., O'Donoghue, D., \& Allen, S. 1985, MNRAS, 212, 9P

Warner, B., O'Donoghue, D., \& Wargau, W. 1989, MNRAS, 238, 73

Williams, G.A., \& Hiltner W.A. 1984, MNRAS, 211, 629

Woudt, P.A., \& Warner, B. 2002, MNRAS, 333, 411 